\title{
Double blind, placebo controlled trial of magnesium carbonate in chronic pyrophosphate arthropathy
}

\author{
MICHAEL DOHERTY, AND PAULA. DIEPPE \\ From the University Department of Medicine, Bristol Royal Infirmary, Bristol
}

Unlike the situation with urate crystals in gout, there is at present no definitive treatment to prevent formation or enhance dissolution of CPPD crystals in patients with pyrophosphate arthropathy. In vitro studies, however, have shown that magnesium exerts a solubilising, growth inhibitory effect on CPPD crystals, ${ }^{1}$ and the possibility that magnesium supplementation might influence CPPD crystals in vivo is suggested by the precipitation of acute pseudogout after lavage of joints with magnesium sulphate ${ }^{2}$ and by the reported benefit of magnesium replacement in a patient with hypomagnesaemia and chondrocalcinosis. ${ }^{3}$ We therefore undertook a six month controlled trial to investigate the therapeutic potential of magnesium in patients with pyrophosphate arthropathy.

A total of 38 patients with chronic pyrophosphate arthropathy (symptomatic arthritis + CPPD crystals in synovial fluid \pm radiographic evidence of chondrocalcinosis) were allocated at random to receive $10 \mathrm{ml}$ thrice daily of either magnesium carbonate $(30 \mathrm{mEq}$ magnesium/day) or placebo. The treatment group (12 men, seven women) and controls (five men, 14 women) were comparable for mean age $(64 \cdot 4 \pm 12$ yr $v 69 \pm 10 \mathrm{yr})$ and duration of disease $(9 \pm 8$ yr $v 8 \pm 5$ yr). Patients were assessed regularly by a single observer and treatment was patient and observer blind.
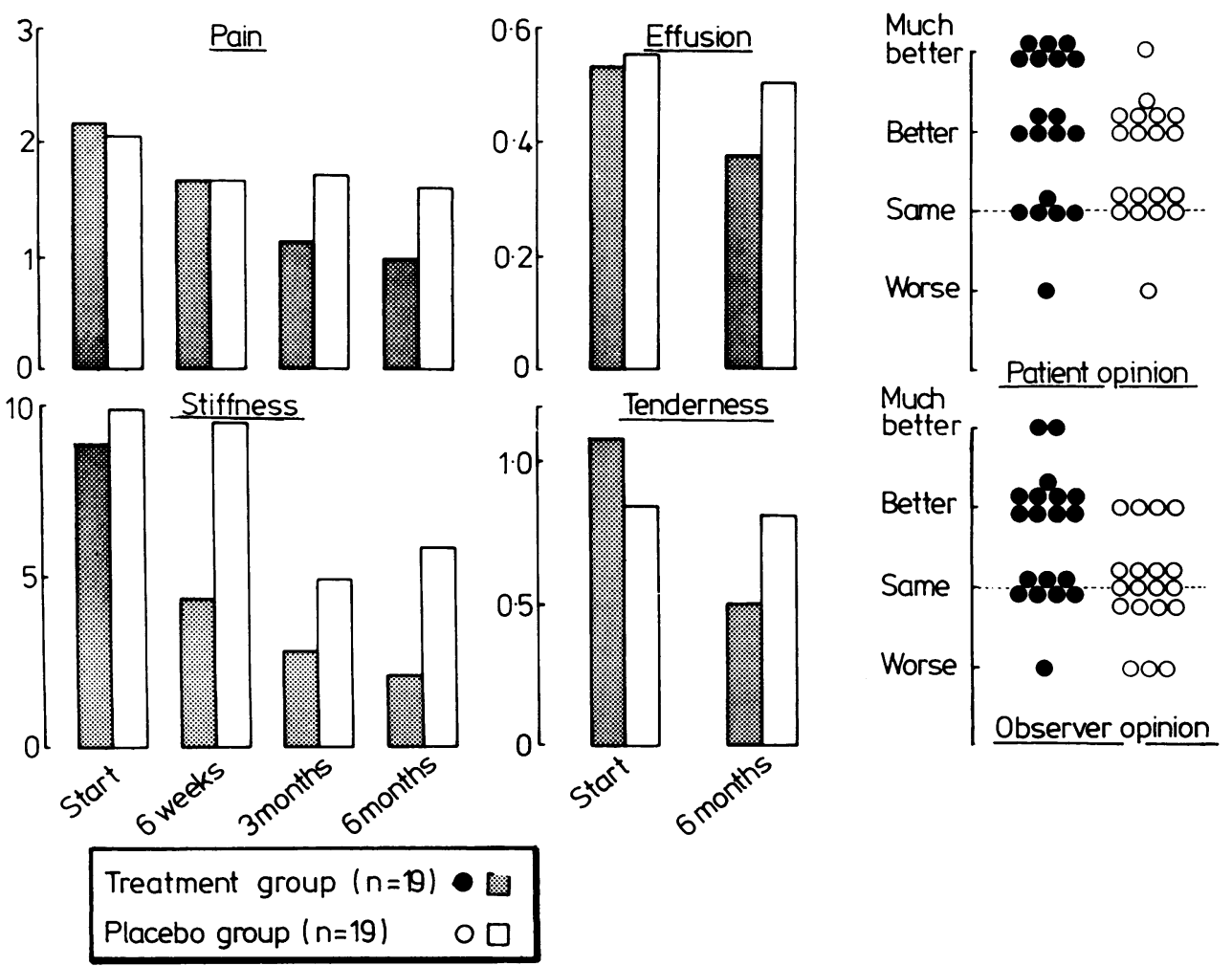

Fig. 1 Duration of pain, effusion, stiffness, and tenderness be fore and after treatment and subjective and objective assessment ofeffect of treatment. Pain was rated on a scale of 0 'none,' 1 'mild,' 2 'moderate,' and 3 'severe,' effusion and tenderness on a scale of 0 'none,' 1 'mild,' and 2 'severe.' Duration of tenderness was measured in minutes. 
Despite a pronounced placebo effect in the controls, the treatment group showed a uniform trend towards improvement in pain, stiffness, effusion (knee), joint line tenderness, and overall subjective and objective assessment (Fig.): at six months improvement over controls was significant for pain $(p<0.05)$ and objective assessment $(p<0 \cdot 05)$. Seven study patients and two controls noted increased bowel frequency; intermittent diarrhoea developed in three (two study patients, one control) but did not necessitate withdrawal from the trial. No changes in biochemical values or synovial fluid were observed apart from an increase in 24 hour urinary magnesium excretion from a mean (SD) of 3.9 $(2 \cdot 1) \mathrm{mmol}(\mathrm{mEq}) / 24 \mathrm{~h}$ to $5 \cdot 7(2 \cdot 5)$ $\mathrm{mmol} / 24 \mathrm{~h}$ in those taking magnesium. The degree of improvement in those taking magnesium did not correlate with renal function, initial magnesium state, or increase in urinary magnesium output. Radiological appearance of chondrocalcinosis did not change over the six month period.

The uniform trend towards improvement in those taking magnesium suggests that further studies of magnesium supplementation are warranted, especially in view of its safety in the elderly patients included in this trial. The pronounced placebo effect we observed serves to emphasise the debilitating nature of pyrophosphate arthropathy and the supportive effect of regular, interested medical attention on patients demoralised by chronic pain and stiffness.

\section{References}

1 Cheng P T, Pritzker K P H. The effect of calcium and magnesium ions on calcium pyrophosphate crystal formation in aqueous solutions. J Rheumatol 1981; 8: 772-82.

2 Bennett R M, Lehr J R, McCarty D J. Crystal shedding and acute pseudogout: an hypothesis based on a therapeutic failure. Arthritis Rheum 1976; 19: 93-7.

3 Runeberg L, Collan Y, Jokinen E J, Lähdevirta J, Aro A. Hypomagnesaemia due to renal disease of unknown aetiology. Am J Med 1975; 59: 873-81.

\title{
Problems encountered in the routine analysis of synovial fluid for crystals
}

\author{
P. R. HEARN, D. F. GUILLAND-CUMMING, AND R. G. G. RUSSELL \\ From the Department of Human Metabolism and Clinical Biochemistry, University of Sheffield Medical School, Sheffield \\ $S 102 R X$
}

Compensated polarised light microscopy is a simple technique that allows identification of crystals by determination of the sign of their birefringence based on colour change with rotation. The technique applied to crystallography of synovial fluid samples was described by Currey and Vernon-Roberts. ${ }^{1}$ Our own diagnostic service was set up in conjunction with a research project on pyrophosphate metabolism and calcium pyrophosphate crystal deposition.

The technique itself is simple if carefully carried out, but there are various problems and pitfalls. We describe some problems that we have encountered and give some hints for those setting up their own diagnostic service.

(1) Microscope. In the absence of a purpose-built polarising light microscope, most micrscopes can be converted by the addition of polarising filters and a first order red compensating filter.

(2) Slides. Ordinary glass microscope slides are used but must be as dust-free as possible; we have noted appreciable amounts of birefringent material on apparently clean slides and coverslips. Slides should be wiped repeatedly with lens tissue. Coverslips are difficult to clean because of their fragility; we rarely use them.

(3) Samples. Synovial fluid aspirates are taken without anticoagulant, as the clots which form trap crystals and considerably help the examination. Whenever possible, samples should be examined the same day, but samples stored at $4^{\circ} \mathrm{C}$ do not appear to alter during storage for up to a week. Storage at room temperature may lead to artefactual crystal growth as reported by Dieppe. ${ }^{2}$

\section{RES U LTS}

In many samples crystals were initially too small to be identified. These were tiny irregular particles or crystals often accompanied by crystals showing a Maltese cross pattern under polarised light. Many of these particles proved to be artefactual. Examination of glove powder currently used by surgeons showed the presence of numerous particles giving the same characteristic Maltese cross appearance. This powder is pure starch, which presumably enters the sample container at the time of aspiration and sample collection. A similar Maltese cross appearance is produced by lipid droplets; these can be dissolved in xylene and similar solvents and are quite commonly seen.

Fragments of cartilage, drawn up at the time of aspiration, may also cause identification difficulties, as may clots of fibrin with cells embedded in them. Irregular, weakly birefringent particles may be hydroxyapatite conglomerates, but we are unable to identify these routinely at present.

Criteria for crystal identification. Clearly, a diagnostic service must have criteria for identification of crystals. A crystal must be: bright under polarised light, exhibit birefringence, or show the required colour change on rotation of the compensating filter. It must look like a crystal-that is, have regular sides (crystal conglomerates are 